Proceedings of the $2^{\text {nd }}$ ICEENG Conference, 23-25 Nov. 1999

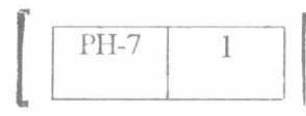
Militart Technical College
Kobry Elkobbah, Cairo, Egypt

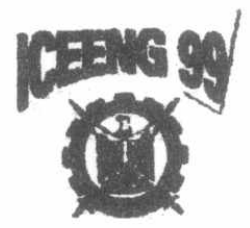

$2^{\text {nd }}$ International conference on Electrical Engineering

ICEENG 99

\title{
ORGANIC CONDUCTORS BASED ON POLY(P-ANISIDINE)
}

\author{
By M.S.Fayed *, M.A.Radwan **, S.R.Gouda***, \\ M.A.Borham ****
}

\section{ABSTRACT:}

Polyansidine organic conductor, was chemically synthesized, via the chemical oxidation of ansidine by persulfate anion in aqueous solution. The prepared polymer was characterized by different methods of analysis such as:

thermogravimetric analysis, electric conductivity, infrared and UV-visible absorption spectroscopy. Polyansidine showed good yield and intermediate electrical conductivity $(\sigma=5 \mathrm{~S} / \mathrm{cm})$. This conductivity assigns it as a semiconductor material. The present work investigates the method of preparation and characterization.

\section{KEY WORDS}

Anisidine, organic conductor, electric conductivity, chemical oxidation, aromatic, amines, and polymerization.

\section{INTRODUCTION}

Over the last decade or so there has been widespread interest in a new class of compounds known as conducting polymers. These polymers produce materials that combine the important electronic and optical properties of semiconductors and metals with specific mechanical properties. The organic polymeric conductors have attracted much scientific and technological interest in recent years. This is due to the large variety of recent and advanced applications in the electronic, photonic based devices and computer systems. The electrical conductivity of these polymers is considered to be intermediate between semiconductors and metals. It ranges between 1-1000 $\mathrm{s} / \mathrm{cm}$ [1]. These properties make them ideal candidates for use in : electronic, capacitors which are used in all almost electronic equipment [2]. Exploitation

$*, * *, * * *, * * * * \ldots$ Egyptian Military Forces 
of organic materials for electrochromic displays (ECDs) was done by several researches. More recently, the use of electroactive organic polymer has been tested widely. Polythiophene and polypyrrole and their analogues have been tested for ECDs use [3]. Currently commercial available capacitors are made of metalized polymer film using metal oxide e,g., $\mathrm{MnO}_{2} \mathrm{O}_{2}$ as cathodes. They have many disadvantages. They are subjected to severe thermal shock owing to high temperature typically $400-600^{\circ} \mathrm{c}$ required for the decomposition of manganese nitrate (III) during manufacture of the capacitor, also the capacitance is not constant beyond $10 \mathrm{kHz}$, and relatively low conductivity which limits the operation of the capacitor above $10 \mathrm{kHz}$. This enhances the failure rate of such capacitor in equipment assembly stage [4]. But using the conductive polymers solves these problems. Recently, conducting polymers have become one of the foci in material science research. This is due to their widespread technological applications. Also : active electrode materials in energy storage [5], opto-electronic devices [6], display devices were extensively searched to be from conducting polymers. These also may be used as chemically modified electrodes [7], and polymer temperature sensors [8]. In addition to the above applications, research and development are continuing in several areas in antistatic applications, sensors, radar blocking applications. , and recently as actuators that do work similar to mechanical-electrical micro machines (MEMs) [9]. In the present work, polyanisidine was selected to be chemically prepared via its oxidation by ammonium Persulfate in an acid medium. The prepared polymer was analyzed and investigated to explore the effect of methoxy-group substituent (p-position in aniline monomer), and consequently discover its useful applications.

\section{EXPERIMENTAL}

\section{Chemicals :}

P-anisidine (Colinbrook), Ammonium persulfate (Aldrich), Ferrous sulphate ( BDH), Hydrochloric Acid (El-Naser), acetone(Prolabo), Formamide (Aldrich), Dimethyl fomamide DMF(Aldrich), benzene(adwic), ethanol (El-Naser), methanol (adwic)

\section{Chemical Polymerization}

P-Anisidine was chemically polymerized by the standard method of chemical oxidation with ammonium persulphate in the presence of hydrochloric acid as follows : Ammonium Persulfate ( $25 \mathrm{~g}$ ) was dissolved in hydrochloric acid $300 \mathrm{ml}, 1 \mathrm{M}$ ) and the solution was cooled to $0^{\circ} \mathrm{C}$ using ice bath. Anisidine $(25 \mathrm{~g})$ was dissolved in hydrochloric acid $(600 \mathrm{ml} 1 \mathrm{M})$. The mixture was stirred for 15 minutes to insure complete solubility and was also cooled to $0^{\circ} \mathrm{C}$. Few 
drops of ferrous sulfate aqueous solution (2.5\%) were added to the anisidine solution. The ammonium Persulfate solution was gradually added to the ansidine solution all over a period of 15 minutes while cooling. After one hour of vigorous stirring of the cold mixture, it was transferred to a freezer for about two hours, a dark brown precipitate was formed, filtered, washed and dried in an oven at $50^{\circ} \mathrm{C}$ for 5 days.

\section{Instrumentation :}

Infrared absorption spectrum was carried out by dispersing samples of the product in $\mathrm{KBr}$ and measured on Perkin-Elmer model 3087 Spectrophotometer, in a wave number range of $500-4000 \mathrm{~cm}^{-1}$. The visible- UV absorption spectrum of the polymer solution was determined using uv-visible Shimmedzu NIR-160 spectrophotometer (Japan). Thermogravimetric analysis (TGA) of the product was carried out with a rate of $150{ }^{\circ} \mathrm{C}$ per minute, the test was conducted in the temperature range of $0-450^{\circ} \mathrm{C}$ using NETZSCH TGZOQ instrument (west Germany). The electrical conductivity was determined using the electronic four point probe Multimeter model 120 Hwellet Packard (France). The test was carried out on a glass substrate with squared shape.

\section{RESULTS AND DISCUSSIONS}

Poly(p-anisidine) was synthesized by the standard method of chemical polymerization of aniline [10], via oxidation of ansidine by ammonium persulfate in aqueous solution of $1 \mathrm{M} \mathrm{HCl}$. In the present polymerization, ammonium persulfate was selected as oxidant, because it was found that it gives a high yeild in aniline oxidation [11]. The weight of polyanisidine product is quite high. Which agrees with our expectation. Since the presence of the electro denotaing methoxy group leads to an easier oxidation compared with aniline. The proposed structure of polyansidine includes quiniod and benzeniod moities in the backbone. The ratios of these two structures depend on the oxidation state occurred. The color was also related to the nature of quinione imine in the backbone. On this base the structure may be significantly modified by doping processes. Which involve the oxidation or reduction of the polymer chain. Consequently, the electric conductivity may be enhanced the redox processes. Functionality, appropriate doping and its level, controlled synthesis and the other factors are the main parameters in these processes. As a result of these chain modification, the polymer can find unique application in advanced technologies. The proposed structure of polyanisidine as a complete reduction form 1 and a completely oxidized form 2, imine and amine and protonated form $\mathrm{s}$ as follows 

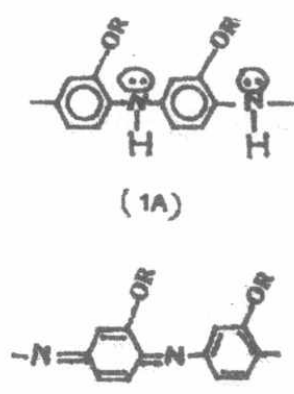

(2A)

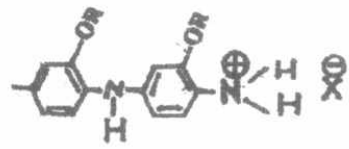

(1s)

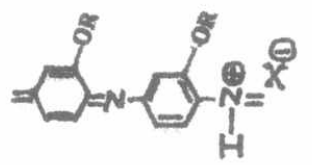

(2s)

The possible mechanism of anisidine polymerization is the loss of an electron from the lone pair to form the corresponding radical cation, which can be represented by the equation :

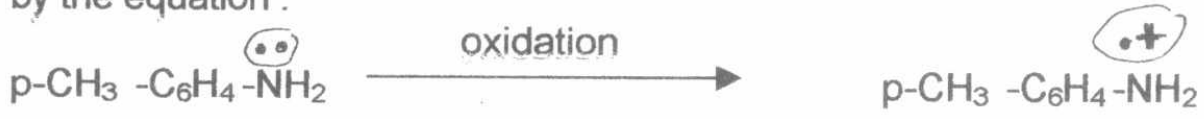

This radical can attack another molecule of anisidine at the para-position. During this attack some structure modification occurs via migration of methoxy group to the o-position to form polyanisidine. The degree of oxidation depends on the molar ratio of oxidizing agent, its time of addition and the type of catalyst used. The polymer weight obtained was $30.4 \mathrm{~g}$, which amounts to $85 \%$ yield.The polymer solubility was tested in different organic solvents. It was found that it is partially soluble in formamide, soluble in dimethyl formamide DMF, and completely insoluble in benzene, ethanol, methanol, and acetone. The electrical resistivity was measured by four point probe multimeter, and electrical conductivity was calculated according to the formula:

$$
\sigma=R \mathbf{D} C / L
$$

$\sigma . . . . .$. conductivity of the polymer film $(\mathrm{S} / \mathrm{cm}$ )

R........resistivity (ohm).

D.......height of the sample.

L...... separation between two silver base poles.

C......the thickness of the polymer film $(\mu \mathrm{m})$.

The electrical conductivity was amounted to $0.56,1.234,1.651 \mathrm{~S} / \mathrm{cm}$ corresponding to three samples prepared with different molar ratios ( monomer I oxidant ) : 1:3, 1:4, 1:5 respectively, and using persulfate in $1 \mathrm{M}$ aqueous hydrochloric acid. These levels of conductivity are less than polyaniline at the same conditions [12]. By comparing the electrical conductivities of the prepared polyaniline and polyanisidine under the same conditions, there was a clear drop in the obtained values. The decrease of the electrical conductivity of 
poly(p-anisidine) compared with polyaniline can be attributed to several factors such as : chemical stucture of the polymer and the extent of oxidation, the conformation of the polymer chain, its stereostructure, the chain packing pattern, the morphology of the polymer in the solid state and the presence of bulky substituents leading to the noncoplanarity of the aromatic rings. The measured conductivity lies in the semiconductor region. This classification according to Fig 1, thus this polymer may be used as a semiconductor material in electronic devices. The role of hydrochloric acid, is the protonation of poly $(p-$ anisidine) to form the corresponding salt, this occurs preferentially at the imine nitrogen $(-\mathrm{N}=)$ as follows:
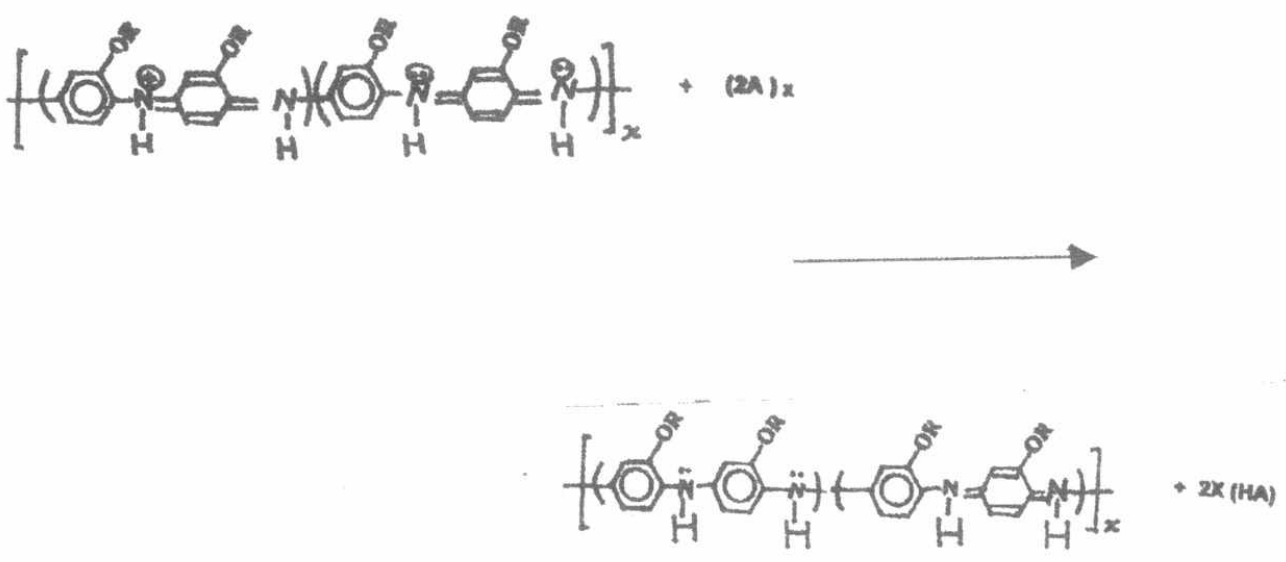

This protonation reaction induce a large increase in the resultant polymer conductivity, since it is known that the totally oxidized form is an insulator, this doping process leads to the formation of a positive charge localized in the polymer chain accompanied by the transformation of aromatic structure towards the quiniod form. Such conductivity can be looked as a result of both the conjugation and regularity of the polymer structure. In the same time the oxidation implies the diffusion of the oppositely charged chloride counterions from the medium to the polymer.

IR-spectrum of the polymer sample was tested and showed in Fig 2 . This spectrum reveals the existance of the bands in the region of $1400-1240 \mathrm{~cm}^{-1}$, which correspond to the $\mathrm{C}-\mathrm{N}$ stretching characteristics of aromatic amines. 
The peak at $1160 \mathrm{~cm}^{-1}$ corresponds to $\mathrm{C}=\mathrm{N}$ in the quinione imine moity. The aromaticity of the polymer can be shown by peaks at $745 \mathrm{~cm}^{-1}$ and 695 $\mathrm{cm}^{-1}$. The $\mathrm{C}-\mathrm{O}-\mathrm{CH}_{3}$ was shown from the peak at $3200 \mathrm{~cm}^{-1}$. The strong band at about $3500 \mathrm{~cm}^{-1}$ region may be attributed to the $\mathrm{N}-\mathrm{H}$ stretching.

\section{UV-visible spectrometry :}

It was reported that UV-visible spectra of the fully reduced form of the parent polymer, polyaniline, lecoemerladine in $\mathrm{N}$-methyl pyrolidone NMP reveals a strong absorption band centered at $340 \mathrm{~nm}$ and a very weak residual absorption at $633 \mathrm{~nm}$. The former band has been assigned to the $\Pi-\Pi^{*}$ transition corresponding to the B-band (benzenoid band). The latter band has been explained to be due to a charge-transfer excitation-like transition from the highest occupied level centered on the benzenoid rings to the lowest unoccupied energy level centered on quinoid rings. It is believed that this excition band can be used as a measure of the oxidation state of polyaniline. As the intensity of excition band decreases relative to $\Pi-\Pi^{*} B$ band, the oxidation state is probably low. The UV-visible spectum of poly(panisidine) shown in Fig 3 shows characteristic bands at $315 \mathrm{~nm}$ and 350 $\mathrm{nm}$. The substitution on benzen rings of methoxy group causes a hypschromic shift of the $\Pi-\Pi^{*}$ transition band from $320 \mathrm{~nm}$ to $315 \mathrm{~nm}$. This band is related to the extent of the conjugation between the adjacent rings in the polymer chain. The resonance energy is at its maximum when the rings are coplanar. So the main effect of the substituent groups of methoxy on the rings, is the destruction of that coplanarity by the steric interference leading to the formation of the stable conformation with certain angle between the planes of the rings. By this way the maximum overlap can not be achieved. The band at $620 \mathrm{~nm}$ in polyaniline disappears in the spectrum of the salt and new peak at $420 \mathrm{~nm}$ was formed.This band was to be due to the charge transfer complex formed that absorp $s$ in the visible region. This band is also shifted to $350 \mathrm{~nm}$ due to the same reason mentioned before. The reported results inspite of its rarity showed that the position of excition band is dependant on the chain length (degree of polymerization) as well as on the distribution of benzeniod and quiniod rings (extent of doping ). The slight difference in absorption bands may be due to the extent of the oxidation and the used solvent.

Thermogravimetric analysis (TGA) Fig 4 for the sample prepared at molar ratio 1:5 (monomer / oxidant) was tested. The polymer shows on weight loss region at $100^{\circ} \mathrm{C}$ with $37.8 \%$ loss in weight. This can be attributed to the removal water. The second weight loss that was located at $295^{\circ} \mathrm{C}$, can be explained by the partial decomposition of the organic polymer. This thermal analysis indicates that the sample is stable up to $290^{\circ} \mathrm{C}$ 
\begin{tabular}{|l|l|l|} 
Proceedings of the $2^{\text {nd }}$ ICEENG Conference, 23-25 Nov. 1999 & PH-7 & 7 \\
\hline
\end{tabular}

\section{CONCLUSION}

Polyanisidine was synthesized successfully by the chemical polymerization of anisidine. via its oxidation by ammonium persulfate in aqueous solution, by employing ferrous sulfate as a catalyst. The characterization of the prepared polymer was done by UV-,IR-spectroscopy, thermalgravimetric analysis, solubility and electrical conductivity. The resultant electrical conductivity was in the range $0.54-1.65 \mathrm{~s} / \mathrm{cm}$, making it very useful as a semiconductor material. From TG analysis. Its clear that the polymer sample is stable up to $290^{\circ} \mathrm{C}$. Due to this thermal stability and its solubility at room temperature (which give an ease processing ), it may be find suitable applications in semiconductor devices.

\section{REFERENCES}

[1] M.Goosey, Speciality polymers, $1^{\text {st }}$ ed. Chapman and Hall, New York, 1987, pag. 85 .

[2] S.P.Sitaram, J.O.Stoffer, and T.J.O.KEEFE, J.of Coating Technology, 69, no 688,1997, p 65.

[3] A.Kitani, Junyano and Kazuosaski, J. of Electroanal.Chem. 1996, 209, 227

[4] S.Sathiyanarayanan et al, Synthetic Metals, 74, 1995, 165-170

[5] T.A.Snothrem (ed). Handbook of Conducting Polymers, volumes 1 and 2. Marcel Decker. New York (1986).

[6] Chao and M.S Wrighton, J.am .Chem .Soc, 109,6627 (1987).

[7] J.Josheph and D.C Trievedt .Bull.Electrochem, 4, 469 (1988).

[8] J.Chem.Phys 1990, 92(4), 2187-88.

[9] Genies.E.M, Boyle.A, Lapkowski.M and T Sintavis. C, Synthetic Metals, 36, 139- 82,1980.

[10] S.Sathiyanarayanan et al, Corrosion Science, volume 33(12), page 183144, 1992.

[11] J.Aber.Science, 246,1989, page 208

[12] S.M.Fayed, M.A. Radwan, S.R.Gouda, M.A.Borham, $8^{\text {th }}$ interenational conference on aerospace science and aviation technology, 4-6 may Egypt, 1999. 


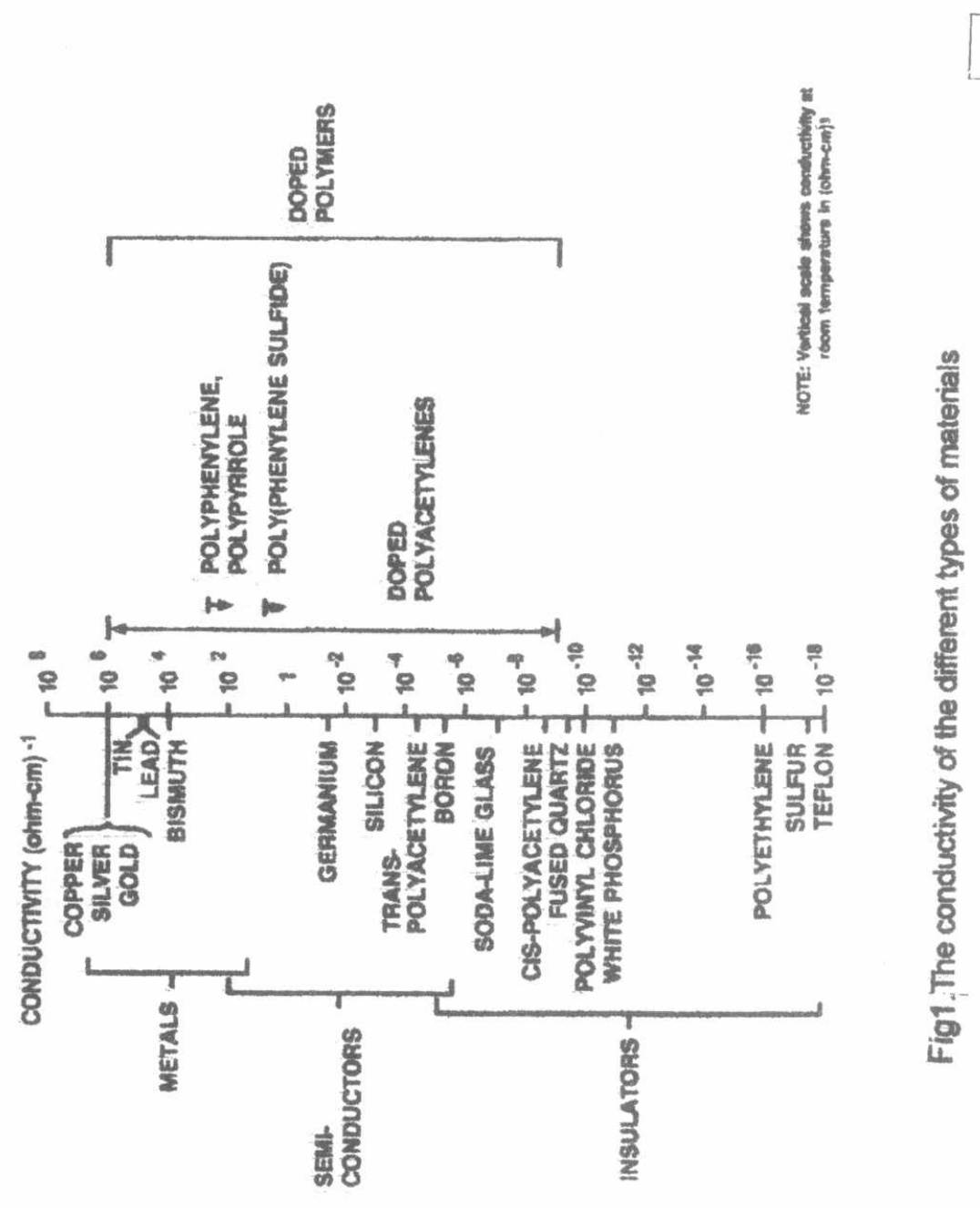




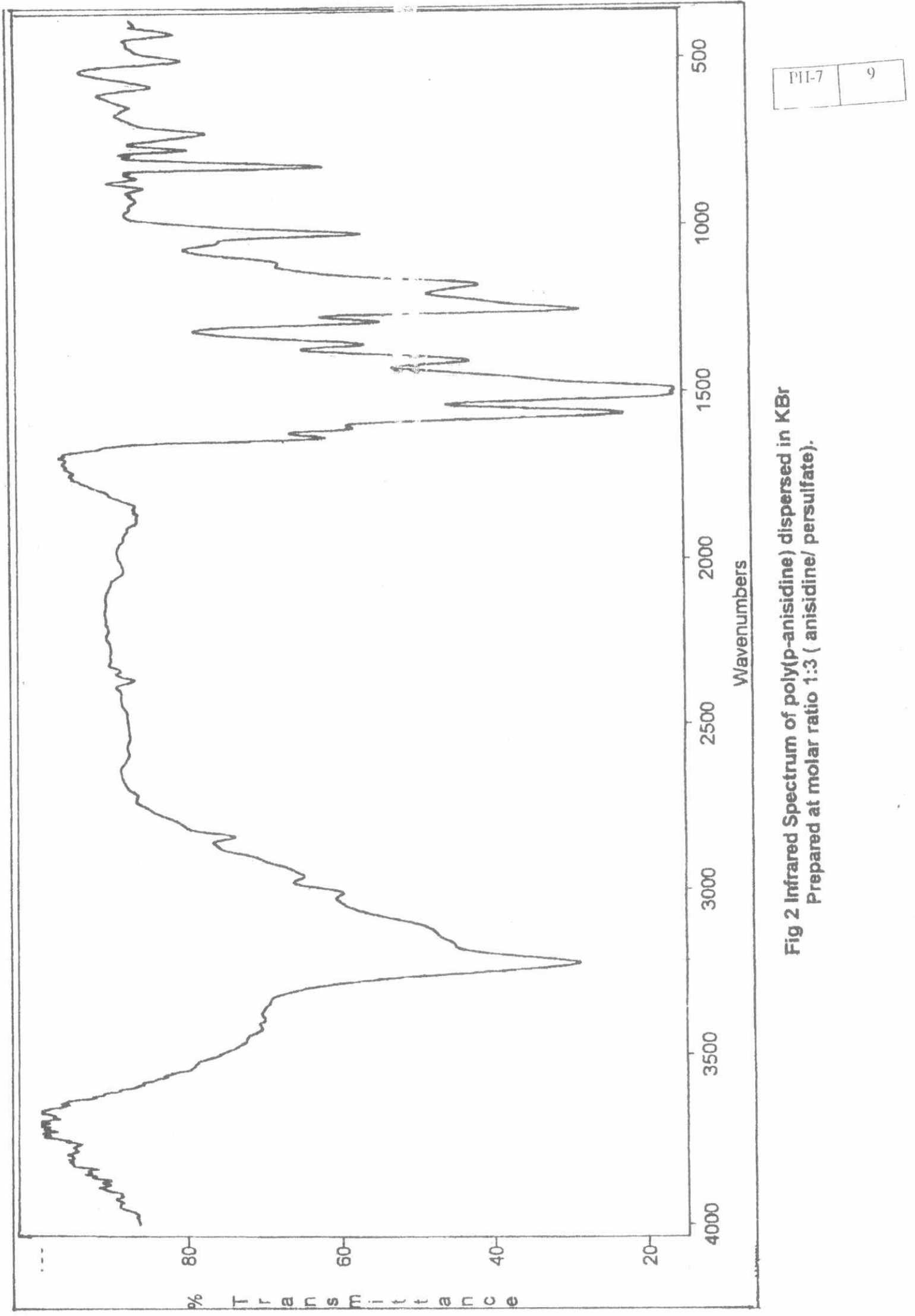




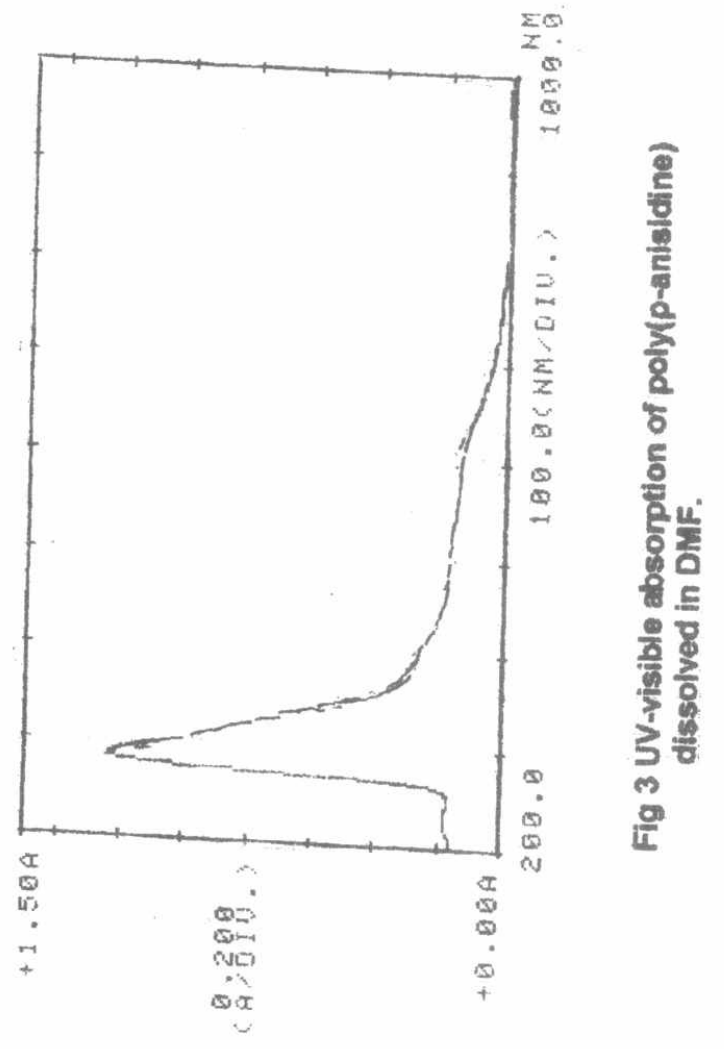



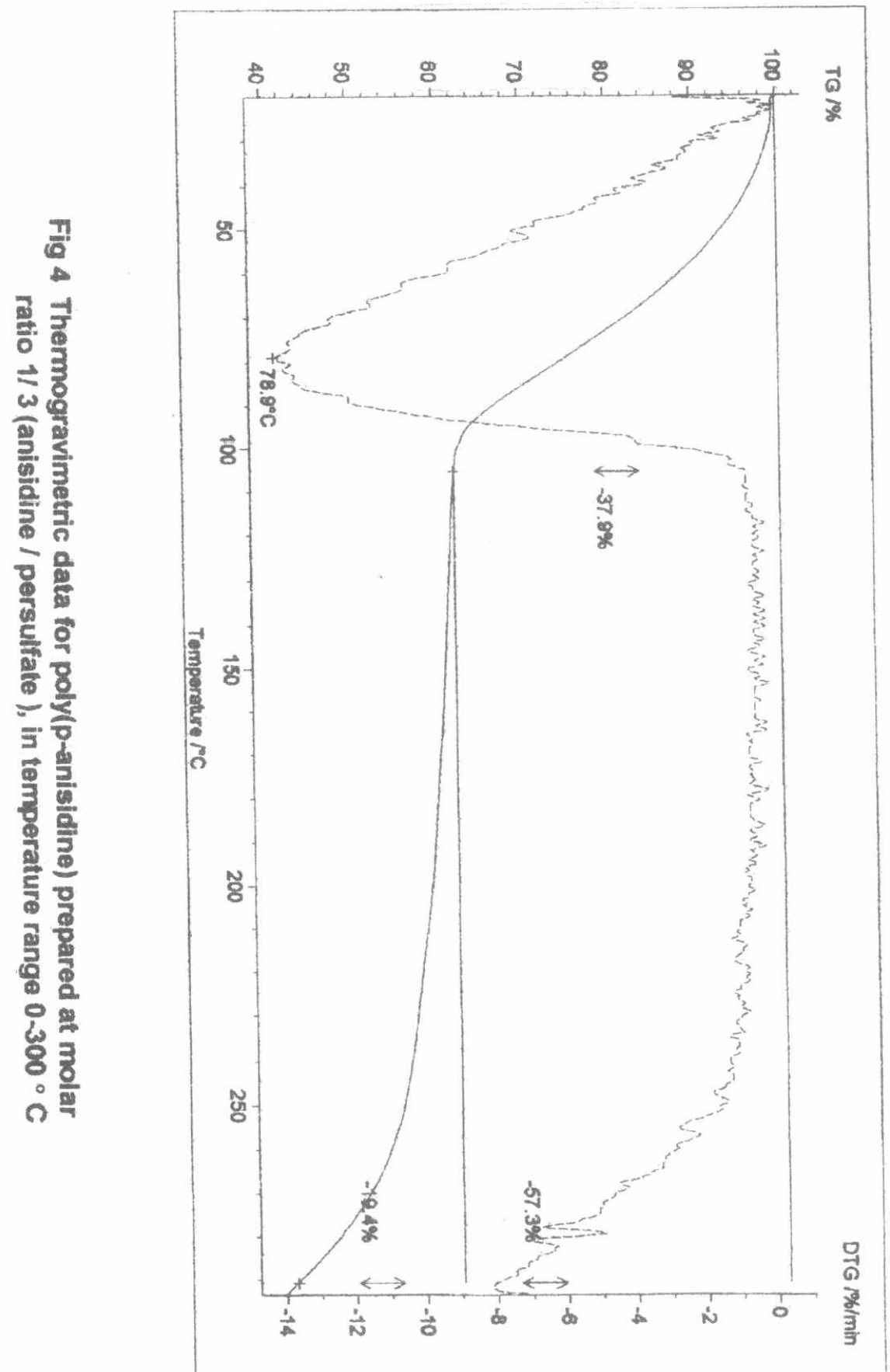

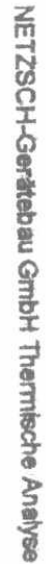

